\title{
RISK FACTORS AFFECTING THE INCIDENCE OF INFECTION AFTER ORTHOPAEDIC SURGERY: THE ROLE OF CHEMOPROPHYLAXIS
}

\author{
Aikaterini Masgala ${ }^{1}$, Efstathios Chronopoulos², Georgios Nikolopoulos ${ }^{3}$, John Sourlas ${ }^{2}$, Stergios Lallos ${ }^{2}$, \\ Emmanuel Brilakis'2, John Lazarettos ${ }^{2}$, Nikolaos Efstathopoulos ${ }^{2}$ \\ ${ }^{1}$ First Department of Internal Medicine, Konstantopoulio General Hospital, Athens, Greece \\ ${ }^{2}$ Second University Orthopaedic Department, Athens Medical School, Konstantopoulio General Hospital, Athens, Greece \\ ${ }^{3}$ Hellenic Centre of Diseases Prevention and Control, Athens, Greece
}

\section{SUMMARY}

The incidence of surgical site infection and urinary tract infection following orthopaedic procedures has diminished in recent years due to modern antimicrobial prophylaxis.

We conducted a case-control study (100 cases, 100 controls) in order to evaluate the risk factors associated with infection after orthopaedic procedures. The following risk factors were defined: gender, age, comorbidities [rheumatoid arthritis, diabetes mellitus, obesity $\left(>30 \mathrm{~kg} / \mathrm{m}^{2}\right)$, peripheral vessel disease], pre- and post-operative glucose levels, pre-operative and post-operative length of stay (days), duration (days) of urinary catheterization, type of parenteral antibiotic prophylaxis (cefotaxime or vancomycin), time of surgery (elective or scheduled), American Society of Anesthesiologists (ASA) Score (0-3), type of surgery (fracture osteosynthesis, joint replacement, spinal surgery, other), and the type of anesthesia administered (general, epidural, spinal).

Urinary tract infection was the most frequent post-surgical infection (71 out of 100 cases) followed by surgical site infection (15 out of 100 cases). Using the multivariable logistic regression model, we found out that only the type of chemoprophylaxis was statistically significant risk factor ( $p<0.001$ ) associated with post-surgical infection. More specifically, the use of vancomycin instead of cephalosporin is associated with a lower risk of infection.

Key words: infection, post-surgical, orthopaedic, risk factors

Address for correspondence: A. Masgala, Konstantopoulio General Hospital, Tmolou 39, Athens 16233, Greece. E-mail: katerina.masgala@ gmail.com

\section{INTRODUCTION}

The incidence of deep and superficial surgical site infection (SSI) and urinary tract infection (UTI) following orthopaedic procedures has extremely decreased during recent decades due to modern antibiotic prophylaxis. Surgical site infection is the most serious complication occurring in $1 \%$ to $3 \%$ of orthopaedic surgical procedures (1). Most of these infections are the result of direct or airborne contamination at the time of surgery although approximately one-third of infections follow hematogenous seeding of the joint. Urinary tract infection is the second most common hospital-acquired infection reaching incidence of $25-28 \%$. Mortality is tripled in patients having UTI and there is evidence of increased risk for metastatic infection around joint replacements although the latter finding remains controversial (2).

Risk factors associated with SSI include obesity, diabetes mellitus, rheumatoid arthritis, increased American Society of Anesthesiologists (ASA) risk index score (3), whereas female sex, number of days of urinary catheterization, and inappropriate catheter management seem to affect the risk of UTI (2).

In order to elucidate further the aetiology of any kind of infection in patients undergoing orthopaedic procedures, we conducted an observational study using a case-control design.

\section{MATERIALS AND METHODS}

A case-control study was performed in a tertiary care university teaching hospital aiming at the identification of risk factors for infection developing after an orthopaedic procedure. The study was approved by the institutional ethics committee.

The study group consisted of patients who experienced an infection following orthopaedic surgery while controls were randomly selected among patients who had undergone orthopaedic surgery without a subsequent infection. The definitions of infections investigated are given below: A superficial surgical site infection had a discharge, which yielded organisms from the culture of aseptically aspirated fluid or tissue from a swab; Deep infection was considered in case of strong clinical and radiological evidence of infection such as periosteal elevation, focal osteolysis, hot and swollen joint, draining sinus and/or positive intraoperative culture; A urinary tract infection was defined as urine culture with $>100,000$ colony-forming units/ml $(\mathrm{CFU} / \mathrm{ml})$ plus urinary symptoms (dysuria, frequency, urgency and/or suprapubic pain and/or fever); Pneumonia was defined as fever plus radiological findings on chest X-ray consistent with pneumonia.

Parenteral antibiotic prophylaxis was started pre-operatively and continued for a maximum of 48 hours after surgery and for 
5-7 days for a potentially contaminated field. Broad spectrum antibiotics (cefotaxime or vancomycin if the patient had a penicillin allergy) were used. The prophylactic anticoagulation regimen consisted of administration of low molecular weight heparin at post-operative day 1 with continuing administration for 6 weeks.

All cases and controls had sterile urine culture pre-operatively. A urinary catheter was inserted perioperatively and was left in place from 12 hours up to 72 hours in several cases.

\section{Statistical Methods}

The descriptive statistics of continuous characteristics are presented as mean \pm standard deviation. The distribution of continuous variables within cases and controls was compared using the Student's t statistic. Categorical characteristics are displayed as frequencies. Potential association between categorical variables was examined by the chi-squared test. The multivariable analysis was conducted using the logistic regression approach. The estimates derived from regression models were in the form of odds ratios (OR), which are presented along with their $95 \%$ confidence intervals $(\mathrm{CI})$.

The effect of the following factors was examined in multivariable analysis: gender, age, comorbidities [rheumatoid arthritis, diabetes mellitus, obesity $\left(>30 \mathrm{~kg} / \mathrm{m}^{2}\right)$, peripheral vessel disease], pre- and post-operative glucose levels, pre-operative and post-operative length of stay (days), duration (days) of urinary catheterization, type of parenteral antibiotic prophylaxis (cefotaxime or vancomycin), time of surgery (elective or scheduled), American Society of Anesthesiologists (ASA) Score (0-3) the ASA score (0-3), type of surgery (fracture osteosynthesis, joint replacement, spinal surgery, other), and the type of anesthesia administered (general, epidural, spinal). In the first step of the model selection strategy, we applied models containing each of the aforementioned variables one at a time. Variables that appeared significant from step one were incorporated concurrently in one model and, subsequently, those that did not remain important were dropped. Insignificant variables from step one were retested in the model derived from step two as they may become important in the presence of others.

All tests of significance were two-sided and a p-value less than 0.05 was indicative of statistical significance. In model selection, the p-value of 0.10 was used as a criterion for inclusion. All statistical analyses were performed using the package STATA 10.0.

\section{RESULTS}

The current study involved 100 cases and 100 controls treated in the orthopaedic department of a tertiary care hospital in Greece during a 4 year period (2005-2009). Among cases, 71 had UTI, 15 had SSI, 10 developed pneumonia, 1 patient had SSI plus UTI while the remaining 3 cases experienced other kind of infection (acute cholecystitis, bacteremia probably related to UTI, and acute sinusitis) (Table 1).

In UTIs, the most common pathogen detected was Escherichia coli (E. coli) (n=32, 45.1\% of UTIs) followed by Pseudomonas aeruginosa (Ps. aeruginosa) (n=14, 19.7\% of UTIs), Enterococcus spp ( $\mathrm{n}=12,16.9 \%$ of UTIs), Klebsiella pneumoniae $(\mathrm{n}=9$, $12.7 \%$ of UTIs), and Acinetobacter spp. (n=4, 5.6\% of UTIs).
Among SSIs, 2 infections (13.3\%) were deep and the rest were superficial. The most common microorganism isolated in SSIs was Staphylococcus aureus (S. aureus) (n=9, 60\% of SSIs) and more than half of these isolates were methicillin resistant (MRSA)

Table 1. Characteristics and clinical parameters of the study population

\begin{tabular}{|l|c|c|c|}
\hline & $\begin{array}{c}\text { Cases } \\
(\mathbf{n}=100)\end{array}$ & $\begin{array}{c}\text { Controls } \\
(\mathbf{n}=100)\end{array}$ & p-value \\
\hline Age & $76.51( \pm 16.76)$ & $66.45( \pm 16.94)$ & $<0.001$ \\
\hline Gender (males) & 23 & 25 & 0.74 \\
\cline { 1 - 2 } Obesity & 44 & 26 & 0.01 \\
\cline { 1 - 2 } Diabetes mellitus & 19 & 14 & 0.34 \\
\cline { 1 - 2 } ASA score & 31 & 62 & \multirow{2}{*}{$<0.001$} \\
\cline { 1 - 3 } 1 & 56 & 35 & \multirow{2}{|l|}{} \\
\cline { 1 - 3 } 2 & 13 & 3 &
\end{tabular}

\begin{tabular}{|l|c|c|}
\hline \multicolumn{3}{|l|}{ Anatomical location of surgery } \\
\hline Knee & 33 & 40 \\
\hline Hip & 15 & 10 \\
\hline Fracture & 45 & 28 \\
\hline Other & 7 & 22 \\
\hline
\end{tabular}

0.004

\begin{tabular}{|c|c|c|}
\hline \multicolumn{3}{|c|}{ Chemoprophylaxis } \\
\hline Cephalosporin & 49 & 28 \\
\hline Vancomycin & 51 & 72 \\
\hline \multicolumn{3}{|c|}{ Type of anesthesia } \\
\hline General & 60 & 73 \\
\hline Epidural & 6 & 8 \\
\hline Spinal & 34 & 19 \\
\hline
\end{tabular}

0.002

Emergency of surgical procedure

\begin{tabular}{|l|c|c|c|}
\hline \multicolumn{1}{|c|}{ Scheduled } & 59 & 72 & 0.05 \\
\hline Urgent & 41 & 28 & \\
\hline $\begin{array}{l}\text { Pre-operation } \\
\text { glucose levels }\end{array}$ & $103.94( \pm 22.16)$ & $100.88( \pm 25.92)$ & 0.37 \\
\hline $\begin{array}{l}\text { Post-operation } \\
\text { glucose levels }\end{array}$ & $109.42( \pm 26.58)$ & $110.19( \pm 31.35)$ & 0.85 \\
\hline $\begin{array}{l}\text { Pre-operation } \\
\text { hospitalization days }\end{array}$ & $3.31( \pm 1.68)$ & $3.67( \pm 2.42)$ & 0.22 \\
\hline $\begin{array}{l}\text { Total duration of } \\
\text { hospitalization }\end{array}$ & $12.35( \pm 3.06)$ & $13.61( \pm 10.09)$ & 0.23 \\
\hline $\begin{array}{l}\text { Days of use of } \\
\text { a urinary catheter }\end{array}$ & $1.26( \pm 1.00)$ & $0.82( \pm 0.73)$ & $<0.001$ \\
\hline
\end{tabular}

\begin{tabular}{|l|c|l|l|}
\hline \multicolumn{5}{|l|}{ Site of infection } \\
\hline Urinary & 71 & & \\
\hline Surgical field & 15 & & \\
\hline Lung & 10 & & \\
\hline SSI+UTI & 1 & & \\
\hline Acute cholecystitis & 1 & & \\
\hline Bacteremia & 1 & & \\
\hline Acute sinusitis & 1 & & \\
\hline
\end{tabular}


( $\mathrm{n}=6,66.6 \%$ of $S$. aureus infections). Other organisms observed were coagulase-negative staphylococci ( $\mathrm{n}=3,20 \%$ of SSIs), E. coli $(\mathrm{n}=1,6.7 \%$ of SSIs), whereas in 2 infections the pathogenic organism remained unidentifiable ( $\mathrm{n}=2,13.3 \%$ of SSIs).

The detailed demographic and clinical characteristics of the participants are presented in Table 1. Participants had a higher mean age at enrollment (76.51 vs. 66.45), were more obese (44 vs. 26), they were assigned higher ASA score, experienced mostly a knee (15 vs. 10$)$ or a fracture surgery ( 45 vs. 28$)$, and they had received mainly cefotaxime as prophylaxis (49 vs. 28).

The univariable logistic regression model revealed the following significant predictors $(\mathrm{p}<0.10)$ of post-operative infection: age, obesity, ASA score, the duration of urinary catheterization, the anatomical location of the surgical procedure, the type of anesthesia and the kind of the chemoprophylactic regimen. However, in multivariable modeling only the type of chemoprophylaxis retained the statistical significance (Table 2). More specifically, cases were almost 60\% less likely (OR: 0.41; 95\% CI: 0.19-0.89) to have received vancomycin as prophylaxis rather than cephalosporin compared with the control population.

\section{DISCUSSION}

Surgical site infection is a rare but true complication in orthopaedic surgery and is the most common compared to others. Although advances in surgical techniques, improvements in operating room ventilation, and the use of prophylactic chemoprophylaxis have all contributed to the decline of SSIs, it still remains the most serious infection following orthopaedic surgery, especially after arhthroplasty.
In our study, SSI represented the second most frequent infection after orthopaedic procedure, a finding not consistent with the results of previous studies where SSI represented the most prevalent post-surgical infection $(4,5)$. Several risk factors have been related to the incidence of SSI following joint arthroplasty including rheumatoid arthritis $(5,6)$, urinary tract infection $(4,7)$, the timing of prophylactic administration of antibiotics $(8,9)$, the use of surgical drainage (10), increased INR (3), admission from a health care facility (1), diabetes mellitus and morbid obesity $\left(>40 \mathrm{~kg} / \mathrm{m}^{2}\right)(5,10,11)$. In the univariable analysis of our study, age, obesity, ASA score, type of surgical procedure, and the kind of chemoprophylactic regimen were significant predictors for infection after orthopaedic surgery and, therefore, for SSI, which was the second most prevalent post-surgical complication in the cases' group.

Urinary infection is the most frequent infective complication in orthopaedic surgery after SSI and represents $28 \%$ of hospital acquired infections. The reported incidence of urinary infection after joint arthroplasty is from $28 \%$ up to $32.6 \%(12,13)$ and approximately $10 \%$ after multilevel spinal instrumentation (14). UTI represented the most frequent of post-operative infections in the most cases included in our study. The use of an indwelling catheter is a well-recognized risk factor for developing UTI. In orthopaedic surgery, urinary retention is common and is attributed to anesthesia, to neural dysfunction of the bladder and, in males, to prostatic hypertrophy $(15,16)$. Thus, an indwelling Foley catheter or intermittent catheterization is often required. Intermittent catheterization has been found to cause less bacteriuria compared with an indwelling catheter for 48 hours (17). On the other hand, short-term use (less than $24 \mathrm{~h}$ ) of an indwelling catheter after joint replacement reduces the incidence of urinary retention without

Table 2. Results of univariable and multivariable logistic regression analysis

\begin{tabular}{|l|c|c|c|c|c|c|}
\hline \multirow{2}{*}{ Variable $^{1}$} & \multicolumn{3}{|c|}{ Univariable analysis } & \multicolumn{3}{c|}{ Multivariable analysis $^{4}$} \\
\cline { 2 - 7 } & OR $^{2}$ & $\left(95 \% \mathrm{Cl}^{3}\right)$ & P-value & OR & $(95 \%$ Cl) & p-value \\
\hline Age & 1.04 & $(1.02,1.06)$ & $<0.001$ & 1.03 & $(0.99,1.06)$ & 0.11 \\
\hline Obesity (Rc: no) & 2.24 & $(1.23,4.06)$ & 0.01 & 2.07 & $(0.90,4.76)$ & 0.09 \\
\hline ASA (Rc:1) & & & $<0.001$ & & & 0.55 \\
\hline 2 & 3.20 & $(1.75,5.85)$ & $<0.001$ & 1.56 & $(0.68,3.58)$ & 0.30 \\
\hline 3 & 8.70 & $(2.30,32.68)$ & $<0.001$ & 2.34 & $(0.22,24.56)$ & 0.48 \\
\hline Days of use of a urinary catheter & 2.07 & $(1.29,3.32)$ & 0.003 & 1.08 & $(0.52,2.23)$ & 0.84 \\
\hline Anatomical location of surgery (Rc: knee) & & & 0.006 & & & 0.22 \\
\hline Hip & 1.82 & $(0.72,4.58)$ & 0.20 & 1.96 & $(0.71,5.42)$ & 0.19 \\
\hline Fracture & 1.95 & $(1.01,3.77)$ & 0.05 & 3.71 & $(0.59,23.53)$ & 0.16 \\
\hline Other & 0.39 & $(0.15,1.01)$ & 0.05 & 0.68 & $(0.20,2.27)$ & 0.53 \\
\hline Chemoprophylaxis (Rc: cephalosporin) & 0.40 & $(0.23,0.73)$ & 0.003 & 0.41 & $(0.19,0.89)$ & 0.03 \\
\hline Type of anesthesia (Rc: general) & & & 0.006 & & & 0.60 \\
\hline Epidural & 0.91 & $(0.30,2.78)$ & 0.87 & 0.79 & $(0.24,2.57)$ & 0.69 \\
\hline Spinal (rax) & 2.18 & $(1.13,4.20)$ & 0.02 & 1.39 & $(0.64,3.03)$ & 0.41 \\
\hline Type of surgery (Rc: scheduled) & 1.79 & $(0.99,3.23)$ & 0.05 & 0.26 & $(0.04,1.74)$ & 0.16 \\
\hline
\end{tabular}

${ }^{1}$ Odds ratios for continuous variables (age and duration of urinary catheterization) correspond to one unit increase

2OR: Odds ratio

${ }^{3} \mathrm{Cl}$ : Confidence interval

${ }^{4}$ The multivariable analysis includes all variables found statistically significant $(p<0.10)$ in univariable modeling

${ }^{5} \mathrm{Rc}$ : reference category 
increasing the rate of urinary tract infection $(16,18)$. HerruzoCabrera et al. showed that the use of a catheter for a period of 1-4 days is associated with a four-fold increase in the incidence of infection while its use for more than 4 days increases the incidence by a factor of 26 (2). UTI might also be connected with other factors. In a previous study, a higher proportion of infected knees was observed in patients who had UTI during the post-operative period although this finding failed to reach statistical significance (7). In a study conducted by Thomas et al. UTI from Ps. aeruginosa in patients who had undergone total hip arthroplasty was attributed to chemoprophylaxis with cephalosporin for more than three days (19). Finally, a high incidence of asymptomatic bacteriuria has been found in patients admitted to the hospital for joint replacement. However, no correlation has been confirmed between bacteriuria and surgical infection in these patients $(20$, $21)$. In the univariate regression model of our analysis, the duration of urinary catheterization ( 1.26 days for cases vs. 0.82 days for controls) along with the type of anesthesia and the kind of chemoprophylactic regimen were significant predictors of infection and, therefore, of UTI. The above-mentioned results are in accord with findings derived from previous studies $(2,14,19)$.

Pneumonia was the third most frequent infection in the cases' group of our study. Pulmonary hypertension (right ventricular systolic pressure $\geq 35 \mathrm{~mm} \mathrm{Hg}$ on transthoracic echocardiography) has been reported as a risk factor for pulmonary complications including pneumonia in orthopaedic surgery (22). Furthermore, earlier surgery has been correlated with low incidence of pneumonia in a study conducted by Simunovic et al. (23). Although none of our patients reported a history of pulmonary hypertension and none had been intubated, 10 out of the 100 cases developed pneumonia. This may be relevant to the advanced age of the cases compared with controls (76.5 years vs. 66.45 years) and to the higher ASA score (56 of the cases have ASA score 2 vs. only 35 controls with ASA score 2), which raises the possibility of aspiration pneumonia.

In multivariable modeling, the type of chemoprophylaxis was the only parameter that retained its statistical significance. In particular, we found that the use of vancomycin is related to significant lower risk of acquiring infection after an orthopaedic procedure. Other studies have also revealed the role of chemoprophylaxis in the development of post-surgical infections. Herruzo-Cabrera et al. have showed that antibiotic prophylaxis decreases the incidence of urinary infection by a factor of 1.91 considering that cefazolin or vancomycin are effective not only for wound infection (24) but also against the habitual contaminants of the urinary tract (2). Suboptimal timing of prophylactic antibiotics was associated with a 3-4 fold increased risk of SSI in a study conducted by Olsen et al. (9). This is similar to the results presented by Classen et al., Kang et al. as well by Takahashi et al., in which postoperative administration chemoprophylaxis had the strongest association with infection $(8,25)$. Finally, a single dose teicoplanin was proved more effective as prophylaxis for total hip or knee arthroplasty compared with multiple doses of broad spectrum antimicrobials (26), a finding which enhances the results of our study.

All observational studies including case-control designs suffer from limitations. In particular, this research considered totally 200 individuals and might lacked power to identify more predictors of post-surgical infections apart from chemoprophylaxis. Second, this study was conducted in one orthopaedic clinic of a tertiary hospital in Athens and thus its results may not be generalized. Finally, all observational studies can be biased or confounded by unmeasured covariates although we tried to take into account all potential risk factors for post surgical infections.

\section{CONCLUSION}

UTI was the most frequent hospital cross infection after orthopaedic procedure in our study followed by SSI. Although many factors were significantly associated with post-operative infection in univariate analysis, only the kind of chemoprophylaxis remained a statistically significant predictor in multivariable modeling. More specifically, the use of vancomycin instead of cephalosporin is associated with a lower risk of infection.

\section{REFERENCES}

1. Lee J, Singletary R, Schmader K, Anderson DJ, Bolognesi M, Kaye KS. Surgical site infection in the elderly following orthopaedic surgery. Risk factors and outcomes. J Bone Joint Surg Am. 2006 Aug;88(8):1705-12.

2. Herruzo-Cabrera R, López-Giménez R, Cordero J, Munuera L. Urinary infection after orthopedic procedures. Int Orthop. 2001;25(1):55-9.

3. Minnema B, Vearncombe M, Augustin A, Gollish J, Simor AE. Risk factors for surgical-site infection following primary total knee arthroplasty. Infect Control Hosp Epidemiol. 2004 Jun;25(6):477-80.

4. Pulido L, Ghanem E, Joshi A, Purtill JJ, Parvizi J. Periprosthetic joint infection: the incidence, timing, and predisposing factors. Clin Orthop Relat Res. 2008 Jul;466(7):1710-5.

5. Chesney D, Sales J, Elton R, Brenkel IJ. Infection after knee arthroplasty a prospective study of 1509 cases. J Arthroplasty. 2008 Apr;23(3):355-9.

6. Poss R, Thornhill TS, Ewald FC, Thomas WH, Batte NJ, Sledge CB. Factors influencing the incidence and outcome of infection following total joint arthroplasty. Clin Orthop Relat Res. 1984 Jan-Feb;(182):117-26.

7. Wilson MG, Kelley K, Thornhill TS. Infection as a complication of total knee-replacement arthroplasty. Risk factors and treatment in sixty-seven cases. J Bone Joint Surg Am. 1990 Jul;72(6):878-83.

8. Classen DC, Evans RS, Pestotnik SL, Horn SD, Menlove RL, Burke JP. The timing of prophylactic administration of antibiotics and the risk of surgical-wound infection. N Engl J Med. 1992 Jan 30;326(5):281-6.

9. Olsen MA, Nepple JJ, Riew KD, Lenke LG, Bridwell KH, Mayfield J, et al. Risk factors for surgical site infection following orthopaedic spinal operations. J Bone Joint Surg Am. 2008 Jan;90(1):62-9.

10. Bachoura A, Guitton TG, Smith RM, Vrahas MS, Zurakowski D, Ring D. Infirmity and injury complexity are risk factors for surgical-site infection after operative fracture care. Clin Orthop Relat Res. 2011 Sep;469(9):2621-30.

11. Edmonston DL, Foulkes GD. Infection rate and risk factor analysis in an orthopaedic ambulatory surgical center. J Surg Orthop Adv. 2010 Fall;19(3):174-6.

12. García-Alvarez F, Al-Ghanem R, García-Alvarez I, López-Baisson A, Bernal M. Risk factors for postoperative infections in patients with hip fracture treated by means of Thompson arthroplasty. Arch Gerontol Geriatr. 2010 Jan-Feb;50(1):51-5.

13. Jinnah RH, Amstutz HC, Tooke SM, Dorey F, Dalseth T. The UCLA Charnley experience: a long-term follow-up study using survival analysis. Clin Orthop Relat Res. 1986 Oct;(211):164-72.

14. West JL 3rd, Ogilvie JW, Bradford DS. Complications of the variable screw plate pedicle screw fixation. Spine (Phila Pa 1976). 1991 May;16(5):576-9.

15. Williams A, Price N, Willett K. Epidural anaesthesia and urinary dysfunction: the risks in total hip replacement. J R Soc Med. 1995 Dec;88(12):699-701.

16. Michelson JD, Lotke PA, Steinberg ME. Urinary-bladder management after total joint-replacement surgery. N Engl J Med. 1988 Aug 11;319(6):321-6.

17. van den Brand IC, Castelein RM. Total joint arthroplasty and incidence of postoperative bacteriuria with an indwelling catheter or intermittent 
catheterization with one-dose antibiotic prophylaxis: a prospective randomized trial. J Arthroplasty. 2001 Oct;16(7):850-5.

18. Carpiniello VL, Cendron M, Altman HG, Malloy TR, Booth R. Treatment of urinary complications after total joint replacement in elderly females. Urology. 1988 Sep;32(3):186-8.

19. Donovan TL, Gordon RO, Nagel DA. Urinary infections in total hip arthroplasty. Influences of prophylactic cephalosporins and catheterization. J Bone Joint Surg Am. 1976 Dec;58(8):1134-7.

20. Glynn MK, Sheehan JM. The significance of asymptomatic bacteriuria in patients undergoing hip/knee arthroplasty. Clin Orthop Relat Res. 1984 May;(185):151-4.

21. Koulouvaris P, Sculco P, Finerty E, Sculco T, Sharrock NE. Relationship between perioperative urinary tract infection and deep infection after joint arthroplasty. Clin Orthop Relat Res. 2009 Jul;467(7):1859-67.

22. Ryu YJ, Chun EM, Shim SS, Kim JS, Kim YH. Risk factors for pulmonary complications, including pulmonary embolism, after total knee arthroplasty (TKA) in elderly Koreans. Arch Gerontol Geriatr. 2010 Nov-Dec;51(3):299-303.

23. Simunovic N, Devereaux PJ, Sprague S, Guyatt GH, Schemitsch E, Debeer J, et al. Effect of early surgery after hip fracture on mortality and complications: systematic review and meta-analysis. CMAJ. 2010 Oct 19;182(15):1609-16

24. Young B, Ng TM, Teng C, Ang B, Tai HY, Lye DC. Nonconcordance with surgical site infection prevention guidelines and rates of surgical site infections for general surgical, neurological, and orthopedic procedures. Antimicrob Agents Chemother. 2011 Oct;55(10):4659-63.

25. Takahashi H, Wada A, Iida Y, Yokoyama Y, Katori S, Hasegawa K, et al. Antimicrobial prophylaxis for spinal surgery. J Orthop Sci. 2009 Jan;14(1):40-4.

26. Kanellakopoulou K, Papadopoulos A, Varvaroussis D, Varvaroussis A, Giamarellos-Bourboulis EJ, Pagonas A, et al. Efficacy of teicoplanin for the prevention of surgical site infections after total hip or knee arthroplasty: a prospective, open-label study. Int J Antimicrob Agents. 2009 May;33(5):437-40.

Received November 28, 2011 Accepted in revised form September 17, 2012 